\title{
Benign Familial Neonatal Convulsions Caused by Altered Gating of KCNQ2/KCNQ3 Potassium Channels
}

\author{
Pasqualina Castaldo, ${ }^{1}$ Emanuele Miraglia del Giudice, ${ }^{2}$ Giangennaro Coppola, ${ }^{3}$ Antonio Pascotto, ${ }^{3}$ \\ Lucio Annunziato, ${ }^{1}$ and Maurizio Taglialatela ${ }^{1}$ \\ ${ }^{1}$ Division of Pharmacology, Department of Neuroscience, School of Medicine, University of Naples Federico II, 80131 \\ Naples, Italy, and ${ }^{2}$ Department of Pediatrics and ${ }^{3}$ Chair of Child Neuropsychiatry, Second University of Naples, 80131 \\ Naples, Italy
}

The muscarinic-regulated potassium current (M-current), formed by the heteromeric assembly of subunits encoded by the KCNQ2 and KCNQ3 genes, is a primary regulator of neuronal excitability; this regulation is accomplished by impeding repetitive firing and causing spike-frequency adaptation. Mutations in KCNQ2 or KCNQ3 cause benign familial neonatal convulsions (BFNC), a rare autosomal-dominant generalized epilepsy of newborns, by reducing the maximal current carried by the M-channels without affecting ion selectivity or gating properties. Here we show that KCNQ2/KCNQ3 channels carrying a novel BFNC-causing mutation leading to an arginine to tryptophan substitution in the voltage-sensing $\mathrm{S}_{4}$ domain of KCNQ2 subunits (R214W) displayed slower opening and faster closing kinetics and a decreased voltage sensitivity with no concomitant changes in maximal current or plasma membrane expression. These results suggest that mutation-induced gating alterations of the M-current may cause epilepsy in neonates.

Key words: benign familial neonatal convulsions; BFNC; muscarinic regulated potassium current; M-current; potassium channel gating; $S_{4}$ voltage sensor; KCNQ2; epilepsy
Voltage-dependent potassium $\left(\mathrm{K}^{+}\right)$channels represent the most heterogeneous class of ion channels with respect to kinetic properties, regulation, pharmacology, and structure (Shieh et al., 2000). In the nervous system, voltage-dependent $\mathrm{K}^{+}$channels play a crucial role in regulating neuronal excitability by controlling action potential duration, subthreshold electrical properties, and responsiveness to synaptic inputs.

The muscarinic-regulated $\mathrm{K}^{+}$current (M-current), first described in peripheral neurons (Brown and Adams, 1980) and subsequently in the CNS (Halliwell and Adams, 1982), is a widespread regulator of neuronal excitability. In fact, during long-lasting depolarizing inputs, this slowly activating and noninactivating current tends to repolarize the neuronal membrane back toward resting membrane potential, thus limiting repetitive firing and causing spike-frequency adaptation (Rogawski, 2000). Receptor-dependent suppression or stimulation of the M-current is therefore a primary mechanism by which neurotransmitters and neuromodulators may enhance or blunt, respectively, neuronal excitability (Marrion, 1997). The molecular identity of the M-channels has remained elusive until the recent demonstration that it is primarily formed by the heteromeric assembly of $\mathrm{K}^{+}$

Received Sept. 10, 2001; revised Oct. 22, 2001; accepted Oct. 25, 2001.

This study was supported by the following grants: Telethon 1058; National Research Council (CNR) 97.01233.PF49, 98.03149.CT04, 99.02614.CT04, 99.00495.PF49, and 01.00804.PF49; Italian Ministry of the University and Scientific and Technological Research (MURST) COFIN 1999 and COFIN 2001 (M.T.); CNR 98.01048.CT04, 98.00062.PF31， 99.02371.CT04， 99.000192.PF31, 01.00169.PF31, and 00.D132-001; MURST and COFIN 2000; and Regione Campania and Instituto Superiore di Sanità (L.A.). We are deeply indebted to Prof. Thomas J. Jentsch (Zentrum für Molekulare Neurobiologie, Hamburg, Germany) for KCNQ2 and KCNQ3 cDNAs.

Correspondence should be addressed to Dr. Maurizio Taglialatela, Division of Pharmacology, Department of Neuroscience, School of Medicine, University of Naples Federico II, Ed. 19, Via Pansini 5, 80131 Naples, Italy. E-mail: mtaglial@unina.it.

Copyright (C) 2002 Society for Neuroscience $\quad 0270-6474 / 02 / 220001-06 \$ 15.00 / 0$ channel subunits encoded by the $K C N Q 2$ and $K C N Q 3$ genes (Wang et al., 1998; Cooper et al., 2000), although other members of the KCNQ subfamily, namely KCNQ4 (Kubisch et al., 2000) and KCNQ5 (Lerche et al., 2000; Schroeder et al., 2000), may contribute to M-current heterogeneity.

The fundamental role played by the M-current in the control of neuronal excitability in humans has received strong genetic support from the discovery that mutations in either KCNQ2 (Biervert et al., 1998; Singh et al., 1998) or KCNQ3 (Charlier et al., 1998) are responsible for benign familiar neonatal convulsions (BFNC), a rare autosomal-dominant idiopathic epilepsy of the newborn. This disease is characterized by the occurrence of multifocal or generalized tonic-clonic convulsions starting at approximately day 3 of postnatal life and spontaneously disappearing after a few weeks or months (Steinlein, 1998). Although neurocognitive development is normal in most BF NC-affected individuals, 10-15\% of them will experience convulsive manifestations later in life (Ronen et al., 1993).

The use of heterologous expression systems to examine the functional effects of the BFNC-causing mutations is crucial for understanding the pathogenesis of the disease and for interpret-

This article is published in The Journal of Neuroscience, Rapid Communications Section, which publishes brief, peerreviewed papers online, not in print. Rapid Communications are posted online approximately one month earlier than they would appear if printed. They are listed in the Table of Contents of the next open issue of JNeurosci. Cite this article as: JNeurosci, 2002, 22:RC199 (1-6). The publication date is the date of posting online at www.jneurosci.org.

http://www.jneurosci.org/cgi/content/full/5989 
ing its dominant mode of inheritance. The results obtained up to today (Biervert et al., 1998; Schroeder et al., 1998; Lerche et al., 1999; Schwake et al., 2000) suggest that most of these mutations cause a mild reduction in the maximal current carried by the KCNQ2/KCNQ3 M-channels, with no significant dominantnegative effects or changes in channel gating or ion selectivity.

In this report, we describe the study of the functional consequences of a novel BFNC-causing mutation in KCNQ2 (c686t) found in a large, four-generation Italian family leading to an arginine to tryptophan substitution in the $\mathrm{S}_{4}$ voltage-sensing domain of KCNQ2 subunits (R214W) (Miraglia del Giudice et al., 2000). The present electrophysiological and biochemical results, showing that the R214W mutation in KCNQ2 altered the gating properties of the M-channels without affecting their maximal current or plasma membrane expression, suggest that mutation-induced gating alterations of the M-current may cause epilepsy in neonates.

\section{MATERIALS AND METHODS}

Isolation of Xenopus oocytes. The dissociation, maintenance, and microinjection of Xenopus oocytes followed standard procedures (Taglialatela et al., 1997). Briefly, ovarian lobes were surgically removed from adult female Xenopus frogs and individual oocytes were dissociated by enzymatic treatment with collagenase (type IA; $2 \mathrm{mg} / \mathrm{ml}$ ) for $45-80 \mathrm{~min}$ in a $\mathrm{Ca}^{2+}$-free solution. In the experiments described in Figure $3 C$, the follicular layer was removed manually (Schwake et al., 2000). Once dissociated, $\mathrm{Ca}^{2+}$ was reintroduced in the oocyte-bathing solution and the oocytes were stored in a $19^{\circ} \mathrm{C}$ incubator for use on the following day.

Mutagenesis and oocyte injection. KCNQ2 and KCNQ3 cDNAs were cloned in pTLN vectors as described previously (Schroeder et al., 1998). Mutations in KCNQ2 were engineered by sequence-overlap extension PCR with the $P f u$ DNA polymerase, using a NotI-PmlI cassette. DNA sequences were verified by manual sequencing. After linearization, plasmids were transcribed in vitro with a commercially available kit (mCAP; Stratagene, La Jolla, CA) using the SP6 RNA polymerase. RNAs were quantified using the RiboGreen RNA quantification kit (Molecular Probes, Leiden, The Netherlands) and stored at $150-250 \mathrm{ng} / \mu \mathrm{l}$ at $-20^{\circ} \mathrm{C}$ in $0.1 \mathrm{M} \mathrm{KCl}$. Xenopus oocytes were microinjected with $50 \mathrm{nl}$ of cRNA diluted to achieve the desired concentration.

Electrophysiology. At 2-7 d after cRNA microinjection, expressed $\mathrm{K}^{+}$ currents were measured at room temperature with the two-microelectrode voltage-clamp technique. The extracellular recording solution contained (in mM) $96 \mathrm{NaCl}, 2 \mathrm{KCl}, 2.6 \mathrm{MgCl}_{2}, 0.18 \mathrm{CaCl}_{2}$, and 5 HEPES, pH 7.5. pClamp software (version 6.0.2; Axon Instruments, Foster City, CA) was used for data acquisition and analysis.

Surface expression of wild-type and mutant KCNQ2 subunits. Surface expression was measured by inserting a hemagglutinin (HA) epitope into the extracellular $\mathrm{S}_{1}-\mathrm{S}_{2}$ linker of Q2 and Q2R214W subunits (Schwake et al., 2000). With expression of the HA-tagged constructs, plasma membrane surface expression was measured by incubating intact oocytes with a rat anti-HA antibody followed by a horseradish peroxidase-coupled goat anti-rat secondary antibody. After $1 \mathrm{~min}$ of incubation in power signal ELISA solution, the chemiluminescence of individual oocytes was quantified with a Lumat LB 9507 luminometer (EG\&G Berthold, Bad Wildbad, Germany) as described previously (Zerangue et al., 1999).

Data analysis and statistics. Activation and deactivation kinetics were calculated by fitting the current records to a sum of two exponential functions, as already reported for KCNQ2/KCNQ3 channels and native M-current (Wang et al., 1998), where $\tau_{\mathrm{f}}$ and $\tau_{\mathrm{s}}$ are the time constants of the fast and slow exponentials, respectively. Conductance-voltage curves were generated by normalizing to the maximal value the tail currents recorded during repolarization to $-70 \mathrm{mV}$ and expressing the normalized values as a function of the preceding voltages. As described for Shaker $\mathrm{K}^{+}$channels (Stefani et al., 1994), the data were fit to a sum of two independent Boltzmann distributions $\left(B_{1}\right.$ and $\left.B_{2}\right)$ of the following form: $y=\max _{1} /\left[1+\exp \left(V_{1}-V\right) / k_{1}\right]+\max _{2} /\left[1+\exp \left(V_{2}-V\right) / k_{2}\right]$, where $V$ is the test potential, $V_{1}$ and $V_{2}$ are the half-activation potentials, $k_{1}$ and $k_{2}$ are the slopes, $\max _{1}$ and $\max _{2}$ are the maximal amplitudes for each Boltzmann distribution, and exp is the exponential function (power of $e$, the base of natural logarithms). IC $_{50}$ values for extracellular TEA $\left(\mathrm{TEA}_{\mathrm{e}}\right)$ blockade were calculated by fitting the percentage of inhibition

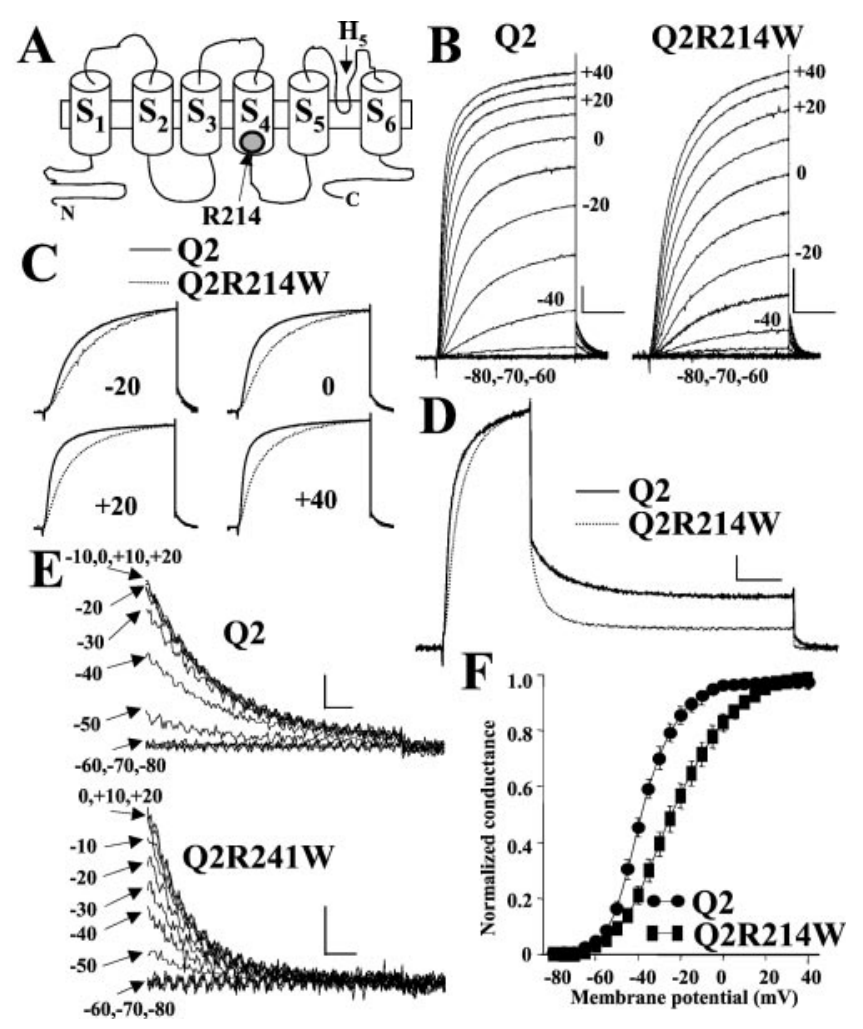

Figure 1. Effect of the R214W mutation on homomeric KCNQ2 channel function. $A$, Putative transmembrane topology of a KCNQ2 subunit. The arginine at position 214 in $\mathrm{S}_{4}$ is indicated by a gray circle. $H_{5}$ denotes the pore region. $B$, Current traces from Xenopus oocytes injected with 5 ng/oocyte of Q2 or Q2R214W cRNAs in response to membrane depolarizations of $10 \mathrm{mV}$ increments from -80 to $+40 \mathrm{mV}$. Holding potential, $-90 \mathrm{mV}$. Calibration: $200 \mathrm{nA}, 500 \mathrm{msec}$. $C$, Comparison between the activation kinetics of Q2 and Q2R214W homomeric channels after appropriate scaling. D, Deactivation kinetics of Q2 and Q2R214W homomeric channels. Holding potential, $-90 \mathrm{mV} ; 1 \mathrm{sec}$ depolarizing pulse to $+20 \mathrm{mV}$, followed by a $3 \mathrm{sec}$ repolarization to $-50 \mathrm{mV}$. Calibration: $10 \%$ of the peak current at $+20 \mathrm{mV}$ (scaling as in $C$ ), $500 \mathrm{msec}$. $E$, Tail currents from Q2 or Q2R214W channels recorded during repolarization to -70 $\mathrm{mV}$ after $1.75 \mathrm{sec}$ depolarizations to the potentials indicated by the arrows. F, Voltage dependence of the normalized conductance of Q2 and Q2R214W channels. The solid lines represent the fits of the experimental data to the sum of two independent Boltzmann distributions (see Materials and Methods).

of the currents elicited by $1.75 \mathrm{sec}$ depolarizations to $+20 \mathrm{mV}$ by the different TEA concentrations (from 0.01 to $30 \mathrm{mM}$ ) to the following binding isotherm: $y=\max /\left(1+X / \mathrm{IC}_{50}\right)$, where $X$ is the $\mathrm{TEA}_{\mathrm{e}}$ (Taglialatela et al., 1991). Statistically significant differences between the data were evaluated with the Student's $t$ test. Data are expressed as the mean \pm SEM.

\section{RESULTS}

\section{Expression of homomeric KCNQ2 R214W channels}

Functional voltage-dependent $\mathrm{K}^{+}$channels assemble as tetramers of identical (homomers) or compatible (heteromers) subunits, each displaying six putative transmembrane segments and a poreforming $\mathrm{H}_{5}$ domain (Shieh et al., 2000). The fourth transmembrane segment $\left(\mathrm{S}_{4}\right)$ contains between four and eight basic residues spaced by hydrophobic amino acids and is thought to form a major part of the voltage sensor (Bezanilla, 2000). Figure $1 \mathrm{~A}$ shows the putative topological arrangement of a single KCNQ2 (Q2) subunit. R214 is the innermost of the six arginine residues in $\mathrm{S}_{4}$ and is highly conserved in the $\mathrm{KCNQ} \mathrm{K}^{+}$channel subfamily; 


\begin{tabular}{|c|c|c|c|c|c|c|c|c|c|c|c|}
\hline \multicolumn{2}{|l|}{ RNA injected } & \multirow[b]{2}{*}{$n$} & \multicolumn{6}{|l|}{ Activation } & \multicolumn{2}{|c|}{ Deactivation } & \multirow{2}{*}{$\begin{array}{l}\text { TEA } \mathrm{e}_{\mathrm{e}} \text { block } \\
\mathrm{IC}_{50}(\mathrm{~mm})\end{array}$} \\
\hline Type & $\begin{array}{l}\text { Amount/oocyte } \\
\text { (ng) }\end{array}$ & & $\tau_{\mathrm{f}}(\mathrm{msec})$ & $\tau_{\mathrm{s}}(\mathrm{msec})$ & $V_{1}(\mathrm{mV})$ & $V_{2}(\mathrm{mV})$ & $\begin{array}{l}k_{1} \\
\text { (mV/e-fold) }\end{array}$ & $\begin{array}{l}k_{2} \\
(\mathrm{mV} / e \text {-fold })\end{array}$ & $\tau_{\mathrm{f}}(\mathrm{msec})$ & $\tau_{\mathrm{s}}(\mathrm{msec})$ & \\
\hline Q2 & 5 & 9 & $61 \pm 4$ & $412 \pm 20$ & $-42.3 \pm 0.5$ & $-24.9 \pm 2.2$ & $6.1 \pm 0.2$ & $8.3 \pm 0.5$ & $121 \pm 14$ & $513 \pm 51$ & $0.13 \pm 0.01$ \\
\hline Q2R214W & 5 & 9 & $154 \pm 26^{*}$ & $639 \pm 43^{*}$ & $-34.9 \pm 0.7^{*}$ & $-9.5 \pm 2.2^{*}$ & $8.3 \pm 0.4^{*}$ & $12.3 \pm 0.6^{*}$ & $84 \pm 7^{*}$ & $354 \pm 38^{*}$ & $0.12 \pm 0.01$ \\
\hline Q2Y284C & 5 & 5 & $67 \pm 6$ & $444 \pm 44$ & ND & ND & ND & ND & ND & ND & $109 \pm 9^{*}$ \\
\hline Q2/Q3 & $0.25 / 0.25$ & 10 & $74 \pm 7$ & $441 \pm 20$ & $-43.6 \pm 0.6$ & $-29.8 \pm 0.9$ & $5.6 \pm 0.1$ & $6.3 \pm 0.2$ & $137 \pm 4$ & $721 \pm 44$ & $1.4 \pm 0.1$ \\
\hline Q2R214W/Q3 & $0.25 / 0.25$ & 9 & $159 \pm 13 \#$ & $1175 \pm 258 \#$ & $-37.3 \pm 0.5 \#$ & $-17.8 \pm 2.3 \#$ & $6.6 \pm 0.3 \#$ & $10.3 \pm 0.5 \#$ & $88 \pm 7 \#$ & $442 \pm 31 \#$ & $1.1 \pm 0.1$ \\
\hline Q2/Q2R214W/Q3 & $0.125 / 0.125 / 0.25$ & 7 & $114 \pm 18 \#$ & $726 \pm 153 \#$ & $-40.6 \pm 0.6 \#$ & $-20.1 \pm 1.1 \#$ & $6.6 \pm 0.4 \#$ & $10.4 \pm 0.6 \#$ & $103 \pm 2 \#$ & $518 \pm 17 \#$ & $1.3 \pm 0.1$ \\
\hline Q2/Q2Y284C/Q3 & $0.25 / 0.25 / 0.5$ & 7 & $83 \pm 5$ & $432 \pm 35$ & ND & ND & ND & ND & ND & ND & $39 \pm 0.3 \#$ \\
\hline
\end{tabular}

$n$, Number of cells recorded; ND, not detected; ${ }^{*} p<0.05$ versus $\mathrm{Q} 2 ; \# p<0.05$ versus Q2/Q3.

the only exception is KCNQ1, where a glutamine residue is present at the corresponding position (Q244).

To assess the functional consequences of the KCNQ2 R214W (Q2R214W) mutation found in the BFNC-affected family, we heterologously expressed the mutant channel subunits in Xenopus oocytes. Homomeric channels composed of mutant Q2R214W subunits displayed activation kinetics much slower than those of homomeric Q2 channels (Fig. 1B,C); at $+20 \mathrm{mV}$, both the fast and slow time constants of activation $\left(\tau_{\mathrm{f}}\right.$ and $\tau_{\mathrm{s}}$, respectively) (Wang et al., 1998) were faster in homomeric Q2 channels with respect to homomeric Q2R214W channels (Table 1). In addition, the ratio of the relative amplitudes of the fast and slow activation components ( $A_{\mathrm{f}}$ and $A_{\mathrm{s}}$, respectively), expressed as $A_{\mathrm{f}} / A_{\mathrm{f}}+A_{\mathrm{s}}$, was greater in Q2 than in Q2R214W homomeric channels (64.5 \pm $3 \%$ versus $49.6 \pm 4.2 \%$, respectively; $p<0.05$ ). Interestingly, the activation kinetics of homomeric channels carrying another mutation found in BFNC-affected families, Y284C, located in the $\mathrm{H}_{5}$ region of KCNQ2 (Q2Y284C), were identical to those of homomeric Q2 channels (Table 1).

Introduction of the $\mathrm{R} 214 \mathrm{~W}$ mutation in $\mathrm{KCNQ} 2$ channels not only slowed the activation kinetics but also increased the rate of channel closing (Fig. 1D,E); in fact, at $-50 \mathrm{mV}$, both the fast and slow deactivation time constants of homomeric Q2R214W were faster than those of Q2 channels (Table 1). In addition, the ratio of the relative amplitudes of the two deactivation components, expressed as $A_{\mathrm{f}} / A_{\mathrm{f}}+A_{\mathrm{s}}$, was $31.4 \pm 2.5 \%$ and $59.9 \pm 4.1 \%$ in $\mathrm{Q} 2$ and Q2R214W homomeric channels $(p<0.05)$. As a result, the percentage of current at the end of the $3 \mathrm{sec}-50 \mathrm{mV}$ pulse relative to the peak current at $+20 \mathrm{mV}$ was decreased from $27.6 \pm 3.4 \%$ in Q2 homomeric channels to $8.6 \pm 1.6 \%$ in Q2R214W homomeric channels $(p<0.05)$. Furthermore, the voltage dependence of homomeric Q2R214W channels was more shallow and shifted toward more depolarized voltages when compared with homomeric Q2 channels (Fig. 1E,F; see below).

\section{Expression of KCNQ2 R214W subunits in heteromeric channels with KCNQ3 or KCNQ2/KCNQ3 subunits}

To mimic the genetic condition of BFNC-affected patients, who carry a single mutated $K C N Q 2$ allele, and considering that KCNQ2 subunits are thought to assemble with KCNQ3 (Q3) subunits to form the M-current (Wang et al., 1998; Cooper et al., 2000), coexpression experiments of Q2R214W subunits with Q3 (1:1 ratio) and with Q2 plus Q3 subunits (0.5:0.5:1 ratio) were also performed.

Similarly to homomeric Q2R214W channels, heteromeric channels composed of Q2R214W/Q3 or Q2/Q2R214W/Q3 subunits displayed slower activation when compared with Q2/Q3
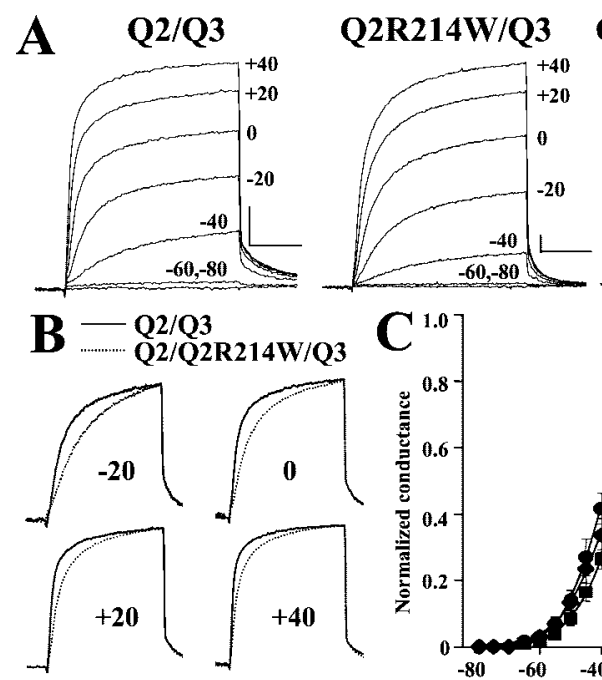

Q2/Q2R214W/Q3
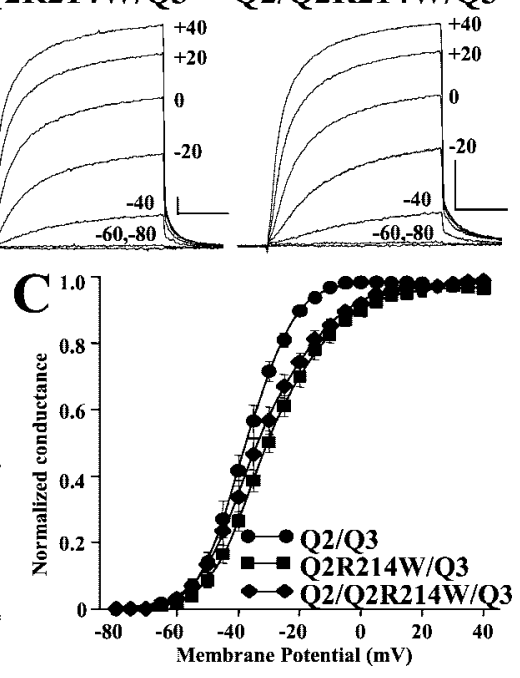

Figure 2. KCNQ2 R214W subunit expression in heteromeric channels with KCNQ3 or KCNQ2/KCNQ3. A, Current traces recorded during depolarization to the indicated voltages of Xenopus oocytes injected with Q2 and Q3 cRNAs $(0.25+0.25 \mathrm{ng} /$ oocyte, respectively); Q2R214W and Q3 cRNAs $(0.25+0.25 \mathrm{ng} /$ oocyte, respectively); or Q2, Q2R214W, and Q3 cRNAs $(0.125+0.125+0.25 \mathrm{ng} /$ oocyte, respectively). Calibration: $100 \mathrm{nA}, 500 \mathrm{msec}$. $B$, Comparison of the activation kinetics of heteromeric Q2/Q3 and Q2/Q2R214W/Q3 channels after appropriate scaling. $C$, Voltage dependence of the conductance of heteromeric Q2/Q3, Q2R214W/Q3, and Q2/Q2R214W/Q3 channels. The solid lines represent the fits of the experimental data to the sum of two independent Boltzmann distributions (see Materials and Methods).

channels (Fig. 2A,B); however, expression of Q2Y284C mutant subunits together with Q2/Q3 (0.5:0.5:1 ratio) failed to affect the Q2/Q3 channel activation kinetics (Table 1). Furthermore, at -50 $\mathrm{mV}$, deactivation $\tau_{\mathrm{f}}$ and $\tau_{\mathrm{s}}$ were slower in Q2/Q3 channels when compared with either Q2R214W/Q3 or Q2/Q2R214W/Q3 channels (Table 1). In addition, the relative weight of the fast deactivation component, expressed as $A_{\mathrm{f}} / A_{\mathrm{f}}+A_{\mathrm{s}}$, was $33.7 \pm 4.4 \%$ for Q2/Q3 channels, $50.6 \pm 1.1 \%$ for Q2R214W/Q3 channels $(p<$ 0.05 vs Q2/Q3), and $49.8 \pm 1.9 \%$ for Q2/Q2R214W/Q3 channels $(p<0.05$ vs Q2/Q3). As a result, the percentage of current at the end of the $-50 \mathrm{mV}$ repolarizing pulse relative to the peak current at $+20 \mathrm{mV}$ was $24.6 \pm 1.2 \%$ in Q2/Q3 channels, $14.3 \pm 2.5 \%$ in Q2R214W/Q3 channels ( $p<0.05$ vs Q2/Q3), and $18.7 \pm 0.4 \%$ in $\mathrm{Q} 2 / \mathrm{Q} 2 \mathrm{R} 214 \mathrm{~W} / \mathrm{Q} 3$ channels $(p<0.05$ vs Q2/Q3). Also, in close analogy to the effects observed in the homomeric configuration, heteromeric assembly of Q2R214W subunits with Q3 or with 
Q2/Q3 subunits decreased the steepness and caused a rightward shift in the voltage dependence of channel activation (Fig. $2 C$; see below).

Interestingly, the gating changes introduced during incorporation of the R214W mutation in KCNQ2 observed in both homomeric and heteromeric configurations were not accompanied by changes in the sensitivity to blockade by $\mathrm{TEA}_{\mathrm{e}}$ (Table 1); in contrast, the Q2Y284C mutation, both in homomeric and heteromeric configurations with Q3 or Q2/Q3 subunits, disrupted the high-affinity binding site for TEA (Taglialatela et al., 1991; Heginbotham and MacKinnon, 1992). Also, the high channel selectivity for $\mathrm{K}^{+}$over $\mathrm{Na}^{+}$was retained with the expression of Q2R214W subunits; in fact, with $2 \mathrm{~mm}$ extracellular $\mathrm{K}^{+}$, the reversal potentials of the expressed currents (in $\mathrm{mV}$ ) were $-92.3 \pm 2.8(n=6),-93.8 \pm 1.3(n=6),-96.6 \pm 2.8(n=5)$, and $-95.6 \pm 2.2(n=5)$ for homomeric Q2, homomeric Q2R214W, heteromeric Q2/Q3, and heteromeric Q2/ Q2R214W/Q3 channels, respectively.

\section{The R214W mutation decreases the voltage dependence of channel activation}

Closer inspection into the voltage dependence of activation of Q2 homomeric channels (Fig. $1 F$ ) and Q2/Q3 heteromeric channels (Fig. $2 C$ ) revealed that the sum of two Boltzmann distributions (Stefani et al., 1994), one with steeper voltage dependence activating at more negative voltages $\left(B_{1}\right)$, and one more shallow activating at more depolarized potentials $\left(B_{2}\right)$, was required to adequately fit the experimental data. The R214W substitution in KCNQ2, expressed in homomeric or heteromeric configurations with Q3 or Q2/Q3 subunits, significantly affected the midpoint potentials $\left(V_{1}, V_{2}\right)$ and the slopes $\left(k_{1}, k_{2}\right)$ of both Boltzmann distributions (Table 1). Quantitatively larger effects of the R214W substitution were observed on the transitions occurring at more depolarized membrane voltages. Comparing the data obtained during triple coexpression of Q2/Q2R214W/Q3 subunits with those of Q2/Q3, we found that the $B_{2}$ component was shifted by 10 $\mathrm{mV}$ toward more depolarized potentials and had a $4 \mathrm{mV} / e$-fold decrease in slope ( $e$ is the base of natural logarithms, and is 2.72.) In contrast, $B_{1}$ was only shifted by $3 \mathrm{mV}$ and had a 1 $\mathrm{mV} / e$-fold decrease in slope. In addition, the relative contributions of the two Boltzmann components $\left(\max _{1}\right.$ and $\max _{2}$ for $B_{1}$ and $B_{2}$, respectively) were $67.7 \pm 5.3 \%$ and $29.4 \pm 5.4 \%$ for homomeric Q2 channels, $48.9 \pm 5.7 \%$ and $50.4 \pm 5.6 \%$ for homomeric Q2R214W channels ( $p<0.05$ vs Q2), $46.6 \pm 5.7 \%$ and $51.9 \pm 5.3 \%$ for heteromeric Q2/Q3 channels, $54.5 \pm 7.2 \%$ and $42.5 \pm 7.4 \%$ for heteromeric Q2R214W/Q3 channels $(p>$ 0.05 vs Q2/Q3), and $54.6 \pm 7.8 \%$ and $43.7 \pm 7.2 \%$ for heteromeric $\mathrm{Q} 2 / \mathrm{Q} 2 \mathrm{R} 214 \mathrm{~W} / \mathrm{Q} 3$ channels $(p>0.05$ vs Q2/Q3).

\section{The KCNQ2 R214W mutation does not reduce the maximal current carried by the M-channels}

Given the significant changes in channel voltage-dependent gating promoted by the KCNQ2 R214W substitution, we studied the effects of this mutation on the maximal current and on the plasma membrane expression of the mutant channel subunits to investigate whether the gating changes observed were the only functional defects induced by the mutation.

The maximal current carried by the channels incorporating Q2R214W subunits in homomeric (Fig. 3A) or heteromeric configuration with Q3 or Q2/Q3 subunits (Fig. 3B) was identical to that carried by homomeric Q2 or heteromeric Q2/Q3 channels, respectively. In contrast, and in agreement with previous studies
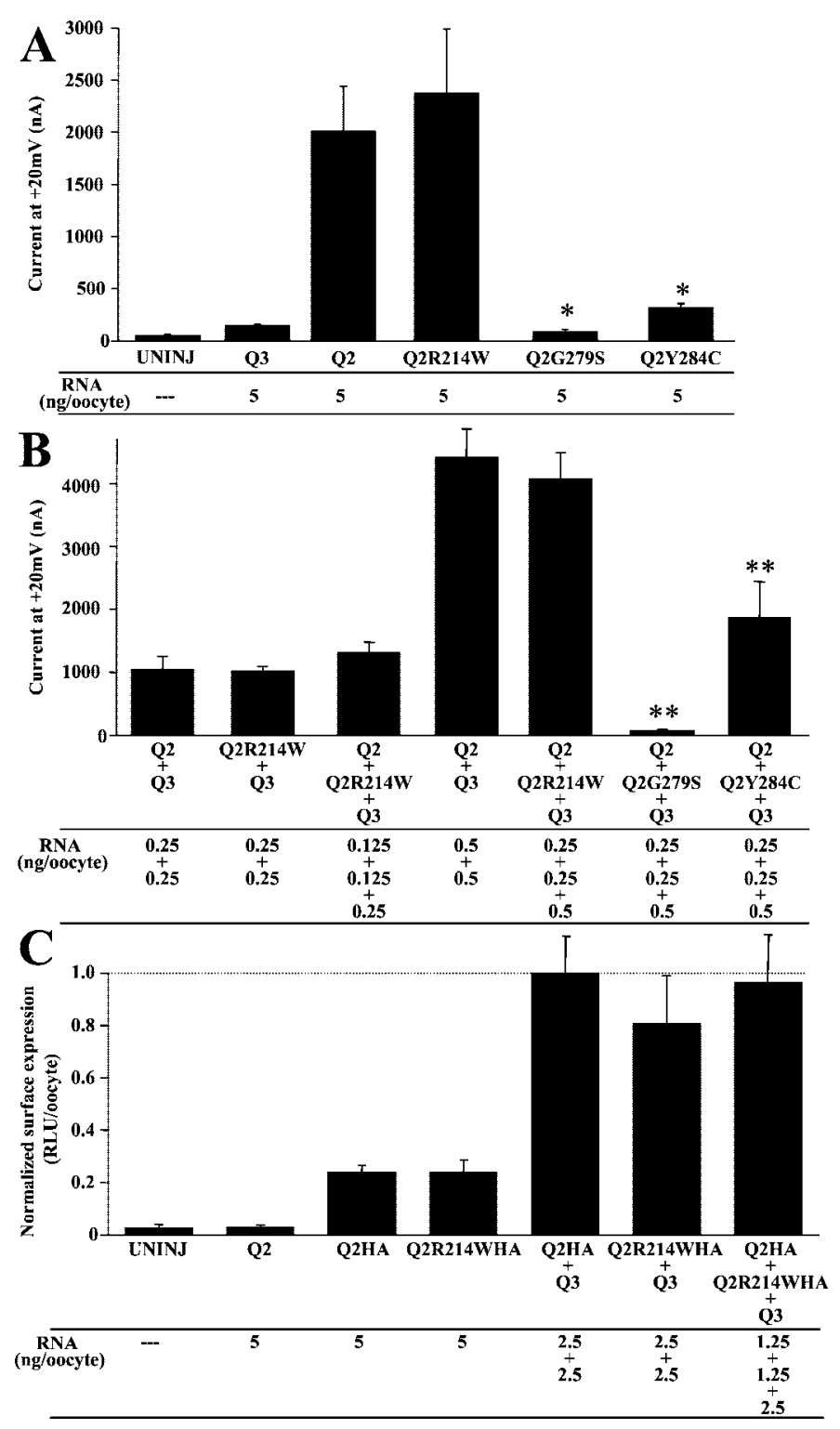

Figure 3. Maximal current and plasma membrane expression of homomeric or heteromeric channels containing KCNQ2 R214W mutant subunits. The maximal current recorded in Xenopus oocytes expressing wild-type Q3, Q2, Q2R214W, Q2G279S, or Q2Y284C subunits in homomeric $(A)$ or heteromeric $(B)$ configurations is shown. Each bar is the mean \pm SEM of the peak current recorded in 10-25 oocytes (three to five batches from different donor frogs) at the end of a $1.75 \mathrm{sec}$ pulse to +20 $\mathrm{mV} .{ }^{*} p<0.05$ versus Q2 (5 ng/oocyte); ${ }^{* *} p<0.05$ versus Q2/Q3 $(0.5+$ $0.5 \mathrm{ng} /$ oocyte, respectively). $C$, Plasma membrane expression of Q2 and Q2R214W subunits. Data are expressed as relative luminescence units $(R L U)$ per oocyte (10 sec reading), after normalization to those of the Q2HA/Q3 group $(15,760,210 \pm 2,750,169$ RLU/oocyte). Each bar is the mean \pm SEM of 12-28 oocytes (three batches from different donor frogs).

(Schroeder et al., 1998), the Y284C BFNC-causing mutation in KCNQ2 reduced the maximal current in both homomeric or heteromeric configurations with Q2/Q3, although to a lesser degree than the strong dominant-negative G279S pore mutation in KCNQ2 (Schroeder et al., 1998; Schwake et al., 2000) (Fig. 3A,B).

Biochemical measurements of surface expression of the mutated subunits (Zerangue et al., 1999; Schwake et al., 2000) showed that the surface levels of KCNQ2 subunits carrying the R214W substitution were identical to those of wild-type KCNQ2 
subunits in both homomeric and heteromeric configurations (Fig. $3 C$ ), a result that is consistent with the lack of effect of the $\mathrm{R} 214 \mathrm{~W}$ substitution on the maximal amount of current carried by channels incorporating the mutated subunits.

\section{DISCUSSION}

Epilepsy is a disorder of recurrent episodes of aberrant hyperexcitability in neuronal networks that affects $\sim 0.5 \%$ of the population (Noebels, 1996). Although convulsive diseases caused by single-gene disorders only account for a minority of idiopathic epilepsies in humans, the identification and the study of the functional consequences of the genetic alterations underlying familiar epilepsies is crucial for clarifying the pathophysiology of the disease, for unraveling the role played by the altered genes, and for designing novel therapeutic approaches (Steinlein, 1998). Among monogenic epilepsies, BFNC represents so far one of the best recognized disease models of generalized idiopathic epilepsies (Hirsch et al., 1999); therefore, investigation of the molecular mechanisms by which the genetic alterations found in affected patients cause BFNC is of fundamental relevance also for the treatment of generalized idiopathic epilepsies in the adult population.

The present study has been performed to elucidate the functional consequences of a novel BFNC-causing missense mutation leading to the replacement of the innermost basic residue with a neutral residue in the $\mathrm{S}_{4}$ voltage-sensing domain of KCNQ2 subunits (R214W) (Miraglia del Giudice et al., 2000). KCNQ2 subunits are crucial (Wang et al., 1998; Cooper et al., 2000) but not exclusive (Kubisch et al., 2000; Lerche et al., 2000; Schroeder et al., 2000) determinants of the M-current, a widely distributed $\mathrm{K}^{+}$-selective current exerting inhibitory control on neuronal excitability.

The results obtained suggest that the primary mechanism for the altered neuronal excitability in BFNC-affected patients carrying the KCNQ2 R214W mutation is a change in the gating properties of the M-channels. In fact, heterologous expression of KCNQ2 R214W subunits, both in homomeric or heteromeric configurations with KCNQ3 or KCNQ2 plus KCNQ3 subunits, led to the appearance of macroscopic currents having slower activation, faster deactivation, and decreased voltage sensitivity. These changes in gating were not accompanied by significant changes in the permeation and blocking properties, because the $\mathrm{K}^{+} / \mathrm{Na}^{+}$selectivity ratio and the sensitivity to $\mathrm{TEA}_{\mathrm{e}}$ block remained unaffected during homomeric or heteromeric expression of KCNQ2 R214W subunits. In contrast, and in agreement with previous studies (Schroeder et al., 1998; Schwake et al., 2000), another mutation found in BFNC-affected families, Y284C, localized in the pore-forming $\mathrm{H}_{5}$ region of $\mathrm{KCNQ}$, failed to affect M-current gating properties, but it abolished channel sensitivity to TEA $_{\mathrm{e}}$ block. The opposite effects exerted on gating and pore properties by the two mutations found in BFNC patients provide strong support for a modular structure of $\mathrm{K}^{+}$channels where the gate and the pore, although functionally coupled, are structurally distinct.

Coexpression experiments also allowed investigation of the molecular basis for the dominant mode of BFNC inheritance. In fact, assuming equal translation capacity of the injected cRNAs (see below), triple coinjection at the 0.5:0.5:1 ratio of KCNQ2 R214W, KCNQ2, and KCNQ3 cRNAs, which is an experimental strategy designed to mimic the heterozygotic condition of BFNCaffected patients, should result in a majority of tetrameric channels carrying a single mutated subunit. The persistence of signif- icant gating alterations in KCNQ2/KCNQ2 R214W/KCNQ3 triple heteromeric channels, when compared with KCNQ2 R214W/KCNQ3 channels (carrying two mutated subunits) or homomeric KCNQ2 R214W channels (having four mutated subunits), indicates that the substitution of the innermost arginine in the $\mathrm{S}_{4}$ voltage sensor of a single KCNQ2 subunit was sufficient to introduce slower rate-limiting steps in the chain of events leading to channel opening (Bezanilla, 2000). This might explain the dominant mechanism of inheritance of BFNC in patients carrying the KCNQ2 R214W mutation.

Previous studies on the functional consequences of the BFNCassociated mutations in KCNQ2 or KCNQ3 have suggested that the $\mathrm{K}^{+}$channel subunits carrying mutations causing extensive deletions in the $\mathrm{C}$ terminus did not form functional channels and did not reach the plasma membrane (Biervert et al., 1998; Schwake et al., 2000); however, subunits carrying missense mutations in the core domain (from $\mathrm{S}_{1}$ through $\mathrm{S}_{6}$ ) can assemble normally but function less efficiently (Schroeder et al., 1998; Lerche et al., 1999; Schwake et al., 2000), as revealed by a 20-40\% maximal current reduction. These results, confirmed by the present experiments with the KCNQ2 Y284C mutation, have led to the conclusion that a mild decrease in M-channel maximal current is a primary pathophysiological mechanism for BFNC caused by missense mutations.

Interestingly, in these studies, the gating properties of the channels carrying the mutant subunits were unaffected. In contrast, the changes in gating described here for the channels incorporating the R214W mutation in KCNQ2 occurred in the absence of modifications in either maximal current or plasma membrane expression. This suggests that changes in M-channel gating may lead to BFNC. In fact, the slower activation and faster deactivation kinetics, coupled with a decreased voltage sensitivity caused by the R214W mutation in the KCNQ2/KCNQ3 heteromeric M-channels, could effectively decrease the ability of this widespread regulator of neuronal excitability to dampen epileptiform discharges in several regions of juvenile brains. The present results are therefore consistent with the idea that the gating alterations prompted by the KCNQ2 R214W mutation cause BFNC because of a decreased neuronal repolarization reserve mediated by the M-current (Rogawski, 2000).

Finally, the altered gating properties induced by the R214W mutation in KCNQ2 might also have considerable implications for genotype-specific therapy. In fact, the novel anticonvulsant molecule retigabine (Main et al., 2000; Wideckenden et al., 2000) shifts the voltage dependence of the M-channels toward more hyperpolarized voltages, a phenomenon opposite to that caused by the R214W mutation investigated here. Thus, it seems likely that the functional consequences of the mutation might influence the antiepileptic efficacy of the drug.

\section{REFERENCES}

Bezanilla F (2000) The voltage-sensor in voltage-dependent ion channels. Physiol Rev 80:555-592.

Biervert C, Schroeder BC, Kubisch C, Berkovic SF, Propping P, Jentsch TJ, Steinlein OK (1998) A potassium channel mutation in neonatal human epilepsy. Science 279:403-406.

Brown DA, Adams PR (1980) Muscarinic suppression of a novel voltage-sensitive $\mathrm{K}^{+}$current in a vertebrate neurone. Nature 283:673-676.

Charlier C, Singh NA, Ryan SG, Lewis TB, Reus BE, Leach RJ, Leppert M (1998) A pore mutation in a novel KQT-like potassium channel gene in an idiopathic epilepsy family. Nat Genet 18:53-55.

Cooper EC, Aldape KD, Abosch A, Barbaro NM, Berger MS, Peacock WS, Jan YN, Jan LY (2000) Colocalization and coassembly of two human brain M-type potassium channel subunits that are mutated in epilepsy. Proc Natl Acad Sci USA 97:4914-4919. 
Halliwell JV, Adams PR (1982) Voltage-clamp analysis of muscarinic excitation in hippocampal neurons. Brain Res 250:71-92.

Heginbotham L, MacKinnon R (1992) The aromatic binding site for tetraethylammonium ion on potassium channels. Neuron 8:483-491.

Hirsch E, de Saint-Martin A, Marescaux C (1999) Benign familial neonatal convulsions: a model of idiopathic epilepsy. Rev Neurol (Paris) 155:463-467.

Kubisch C, Schroeder BC, Friedrich T, Lutjohann B, El-Amraoui A, Marlin S, Petit C, Jentsch TJ (2000) KCNQ4, a novel potassium channel expressed in sensory outer hair cells, is mutated in dominant deafness. Cell 96:437-446.

Lerche C, Scherer CR, Seebohm G, Derst C, Wei AD, Busch AE, Steinmeyer K (2000) Molecular cloning and functional expression of KCNQ5, a potassium channel subunit that may contribute to neuronal M-current diversity. J Biol Chem 275:22395-22400.

Lerche H, Biervert C, Alekov AK, Schleithoff L, Lindner M, Klinger W, Bretschneider F, Mitrovic N, Jurkat-Rott K, Bode H, Lehmann-Horn F, Steinlein OK (1999) A reduced $\mathrm{K}^{+}$current due to a novel mutation in KCNQ2 causes neonatal convulsion. Ann Neurol 46:305-312.

Main MJ, Cryan JE, Dupere JR, Cox B, Clare JJ, Burbidge SA (2000) Modulation of KCNQ2/3 potassium channels by the novel anticonvulsant retigabine. Mol Pharmacol 58:253-262.

Marrion NV (1997) Control of M-current. Annu Rev Physiol 59:483-504.

Miraglia del Giudice E, Coppola G, Scuccimarra G, Cirillo G, Bellini G, Pascotto A (2000) Benign familial neonatal convulsion (BFNC) resulting from mutation of the KCNQ2 voltage sensor. Eur J Hum Genet 8:994-998.

Noebels JL (1996) Targeting epilepsy genes. Neuron 16:241-244.

Rogawski MA (2000) KCNQ2/KCNQ3 $\mathrm{K}^{+}$channels and the molecular pathogenesis of epilepsy: implications for therapy. Trends Neurosci 23:393-398.

Ronen GM, Rosales TO, Connolly M, Anderson VE, Leppert M (1993) Seizure characteristics in chromosome 20 benign familial neonatal convulsions. Neurology 43:1355-1360.

Schroeder BC, Kubisch C, Stein V, Jentsch TJ (1998) Moderate loss of function of cyclic-AMP-modulated $\mathrm{KCNQ} 2 / \mathrm{KCNQ3} \mathrm{K}^{+}$channels causes epilepsy. Nature 396:687-690.

Schroeder BC, Hechenberger M, Weinreich F, Kubisch C, Jentsch TJ (2000) KCNQ5, a novel potassium channel broadly expressed in brain, mediates M-type currents. J Biol Chem 275:24089-24095.

Schwake M, Pusch M, Kharkovets T, Jentsch TJ (2000) Surface expression and single channel properties of KCNQ2/KCNQ3, M-type $\mathrm{K}^{+}$ channels involved in epilepsy. J Biol Chem 275:13343-13348.

Shieh C-C, Coghlan M, Sullivan JP, Gopalakrishnan M (2000) Potassium channels: molecular defects, diseases, and therapeutic opportunities. Pharmacol Rev 52:557-593.

Singh NA, Charlier C, Stauffer D, DuPont BR, Leach RJ, Melis R, Ronen GM, Bjerre I, Quattlebaum T, Murphy JV, McHarg ML, Gagnon D, Rosales TO, Peiffer A, Anderson VE, Leppert M (1998) A novel potassium channel gene, $\mathrm{KCNQ} 2$, is mutated in a inherited epilepsy of newborns. Nat Genet 18:25-29.

Stefani E, Toro L, Perozo E, Bezanilla F (1994) Gating of Shaker K ${ }^{+}$ channels. I. Ionic and gating currents. Biophys J 66:996-1010.

Steinlein OK (1998) New insights into the molecular and genetic mechanisms underlying idiopathic epilepsies. Clin Genet 54:169-174.

Taglialatela M, Vandongen AM, Drewe JA, Joho RH, Brown AM, Kirsch GE (1991) Patterns of internal and external tetraethylammonium block in four homologous $\mathrm{K}^{+}$channels. Mol Pharmacol 40:229-307.

Taglialatela M, Castaldo P, Iossa S, Pannaccione A, Fresi A, Ficker E, Annunziato L (1997) Regulation of the human ether-a-gogo related gene (HERG) $\mathrm{K}^{+}$channels by reactive oxygen species. Proc Natl Acad Sci USA 94:11698-11703.

Wang HS, Pan Z, Shi W, Brown BS, Wymore RS, Cohen IS, Dixon JE, McKinnon D (1998) KCNQ2 and KCNQ3 potassium channel subunits: molecular correlates of the M-channel. Science 282:1890-1893.

Wideckenden AD, Yu W, Zou A, Jegla T, Wagoner PK (2000) Retigabine, a novel anticonvulsant, enhances activation of KCNQ2/3 potassium channels. Mol Pharmacol 58:591-600.

Zerangue N, Schwappach B, Jan YN, Jan LY (1999) A new ER trafficking signal regulates the subunit stoichiometry of plasma membrane K(ATP) channels. Neuron 22:537-548. 